\title{
Maternal competencies among mothers with infants at early infancy in Sri Lanka; a qualitative evaluation
}

\author{
Karunanayaka ADSS ${ }^{1}$, Wijesinghe $\mathrm{CJ}^{2}$, Somasiri KG ${ }^{3}$ \\ ${ }^{\prime}$ Department of Nursing, Faculty of Allied Health Sciences, University of Ruhuna, Galle, Sri Lanka. \\ Departments of ${ }^{2}$ Community Medicine, ${ }^{3}$ Physiology, Faculty of Medicine, University of Ruhuna, Galle Sri Lanka. \\ Correspondence: Ms.ADS S Karunanayaka \\ e-mail: shashika_karunanayaka@yahoo.com \\ (D) https://orcid.org/0000-0002-4937-0884
}

\begin{abstract}
Introduction: Maternal competencies among mothers with infants at early infancy are sparsely described in the literature. This study explores the dimensions of competencies among mothers with infants at early infancy in the Sri Lankan context.
\end{abstract}

Methods: An exploratory qualitative study was conducted using focus group discussions with mothers, fathers, and grandparents of infants aged up to 6 months and in-depth interviews with health personnel providing maternal and child health services for these mothers and infants. The Framework approach for qualitative data analysis was used to identify dimensions of parenting competencies in mothers at early infancy.

Results: Five dimensions of maternal parenting competencies were identified i.e.; feeding the infant, maintaining hygiene, dealing with crying, dealing with illnesses and recognizing the normal growth and development.

Conclusions: The findings can be used to develop culturally specific tools to assess the level of parenting competencies in mothers and to design maternal educational interventions to reinforce their parenting skills.

Keywords: Early infancy, maternal competencies, parenting, qualitative study, Sri Lanka

\section{Introduction}

The birth of a child is not only a time of change in the life of a family but also a critical event characterised by personal, marital and social reorganisation (1-3). Pregnancy, childbirth, and parenting are normal momentous life events for women and their families. Thus, childbirth and parenting are a major concern in maternal, child, and family healthcare.

Assessment of parenting competencies is very crucial to ensure the health and well-being of the child. It helps to understand the characteristics and patterns of a parent's functioning and child-rearing roles. Parenting competencies are defined as ' $a$ set of knowledge, skills, and attitudes that facilitate and optimize the performance of the parental role with the necessary mastery to ensure the maximum potential for the child's growth and development' (4). According to the International Council of Nurses (5), parenting involves four dimensions: taking on responsibilities for the effective exercise of the role; optimizing the child's growth and development; incorporating the child into the family, and behaving according to the expected behaviours of being a mother and father. The mastery of parenting competencies influences how each parent interprets his or her behaviour and his or her child's behaviour (6). Therefore, the acquisition of knowledge and skills related to the child's needs are promoted with the acquisition and development of parenting competencies, with the subsequent improvement of confidence, satisfaction and mastery in-role performance (4). 
The most rapid period of a child's growth and development or the "critical window" of life is considered as before birth and during the first two to three years of life (2). This period has the greatest impact on the child's future growth and development and is critical for setting the foundation for all later well-being (3). Studies reveal that all children need protection and care during the first months of life to support all aspects of their growth and development (physical, social, emotional, cognitive and spiritual) and parents are the best persons to provide such care with quality parenting competencies (7). The first few months of life or the early infancy have many dependency needs which include adequate feeding, adequate hydration, adequate cleaning, adequate shelter, and more specifically, keeping the body temperature within the narrow range, and warmth and affection (7). It was highlighted that the greatest number of dependency needs seem to be encompassed in early infancy, and dependency needs begin to change and decrease with age and maturity (8). Therefore, the acquisition of parenting competencies is crucial for parents with infants at early infancy.

The assessment and identification of care needs is the first step of any intervention (5). Hence, the assessment of the parenting competencies is a relevant dimension of maternal and child care plans by the service providers (4).

The Demographic and Health Survey, Sri Lanka, $2006 / 07$ revealed that $6.4 \%$ of women had begun childbearing when they were teens (9). The reproductive health information database of the Family Health Bureau of the Ministry of Health, Sri Lanka, indicates that $6.5 \%$ of the total pregnancies registered during the year 2010 were teenage pregnancies (10). These teen parents face many social and economic problems related to parenting (11). Similarly, they may lack competencies in child-rearing (12).

In Sri Lanka, maternal education sessions on certain parenting competencies such as breastfeeding and maintaining the hygiene of the infant are conducted as a routine practice during pregnancy at the field Maternal and Child Health (MCH) clinics and during home visits in the antenatal and postnatal period by Public Health Midwife (PHM). However, a proper evaluation of the parenting competencies of the mothers is not done by the $\mathrm{MCH}$ services to assess the effectiveness of these sessions and to identify the areas needing attention.

This study was designed to explore the perceptions of a group of parents, grandparents, and providers of maternal and child health care services regarding parenting competencies required by the mothers with infants at early infancy to identify main dimensions and specific knowledge and skills. The findings will be used as a preliminary step of developing a tool for assessing parenting competencies and will also be useful in designing parenting education interventions for new mothers to enhance their competencies.

\section{Methods}

This study was conducted as a preliminary step of a larger study aiming to develop and validate a culturally specific assessment instrument to assess the parenting competencies among mothers with infants at the early infancy period in Sri Lanka. To generate items for the instrument, an extensive exploration of various concerns on parenting competencies among mothers with infants at early infancy was conducted between September and December of 2017, using two qualitative methods; focus group discussions (FGD) and in-depth interviews (IDI).

FGDs were conducted with mothers, fathers, and grandparents of infants at the early infancy period registered in $\mathrm{MCH}$ clinics in Matara District, Sri Lanka. Matara district was selected as the study setting for FGDs to avoid data contamination during the main study that would be conducted in Galle district. IDIs were conducted with $\mathrm{MCH}$ care providers in two Teaching Hospitals, field $\mathrm{MCH}$ clinics, and a general practice in Galle District, Sri Lanka considering the convenience of conducting interviews at the familiar field to the principal investigator.

Participants for the FGDs were selected based on non-probability convenience sampling. Infants aged up to 6 months registered at the MCH clinics were identified and their parents and grandparents were recruited through public health staff by considering the eligibility criteria. Mothers who had given birth to multiple or pre-term babies and those having 
physical or mental disabilities, mothers of infants with any medical or surgical complications at birth or afterward and those who require special care, mothers who live outside the Matara district and who attend the clinics only temporarily were excluded from the study. The mothers who cannot communicate in Sinhala (the medium used for FGD) were also excluded. A total of nine FGDs with eight participants per session were conducted with mothers, fathers, and grandparents (three FGDs per each group).

For IDIs, a pediatrician, a general practitioner, two Medical Officer of Health, two Public Health Nursing Sisters, two PHMs, and two senior nursing officers working in the pediatric and postnatal wards were selected and recruited based on service experiences with postnatal mothers and infants at early infancy. Service providers who had difficulty in assigning a time slot for IDIs due to busy schedules and those without a genuine interest to take part in the study were excluded.

Data collection was initiated after obtaining prior approval from the health authorities. Written informed consent was obtained from the participants after explaining the purpose of the study. FGDs were conducted by the principal investigator as the moderator and note-taking and audio recording were done by a trained research assistant. The discussions were conducted in a separate room at the local $\mathrm{MCH}$ clinics at a convenient time to ensure maximum participation. Each discussion lasted between 30 and 45 minutes. IDIs with service providers were conducted by the principal investigator at their respective workplaces after a prior appointment. Each interview lasted between 45 minutes to one hour and was audio recorded by the principal investigator.

A pre-tested guide with semi-structured and structured questions was used to preserve the uniformity of the FGDs and IDIs. Data were collected until the data saturation was achieved. A checklist was used in every session to maintain the completeness of the data collection procedure to enhance the quality of data collection. Only one session was conducted on a given day. After each session, participants were given a summary of the outstanding facts made and they were allowed to volunteer any additional information to obtain a more comprehensive view.
All the discussions were conducted in Sinhala language and notes were taken down during the interviews were completed in detail immediately afterward by the principal investigator to ensure the accuracy of information. FGD and IDI recordings were transcribed verbatim.

The Framework approach for qualitative data analysis (13) was employed in the analysis. Interview transcripts were transcribed verbatim and the research team got familiarised with the whole interview by re-listening to the recordings. The transcripts were read carefully to obtain a holistic impression and to identify the codes. The final set of codes was selected to form the analytical framework with the agreement of the research team. Subsequently, the data were charted and a matrix was developed manually, with the inclusion of illustrative quotations. The summarised data sheet was used to identify the characteristics and patterns of data concerning the experiences and perceptions of parenting competencies.

Ethical clearance was obtained from the Ethics Review Committee of the Faculty of Medicine, the University of Ruhuna, Sri Lanka (Reference No:19/12/2016:3.4). Participants were recruited voluntarily and were given the freedom to withdraw from the study or to refuse participation without prejudice. Informed written consent was obtained from all participants. Strict confidentiality of information was maintained and participants were identified using serial numbers and no names were mentioned in the notes or audio recordings. The study findings were presented in a generalised form.

\section{Results}

A total of 24 participants of mothers, fathers, and grandparents were included for FGDs, whereas 10 service providers were recruited for the IDIs.

Five themes emerged from the analysis describing five major dimensions of parenting competencies; namely, (1) feeding the infant, (2) maintaining hygiene, (3) dealing with crying, (4) dealing with illnesses and (5) recognising the normal growth and development. Verbatim quotes from the study participants are labeled as follows; mothers (Mo), fathers $(\mathrm{Fa})$, grandparents $(\mathrm{Gp})$ and service providers $(\mathrm{Sp})$. 


\section{Theme 1. Feeding the infant}

The study found that infant feeding is a mainstay among all the dimensions of parenting competencies required by mothers with infants at early infancy as infants are entirely dependent on the mother during this period. Breastfeeding was unanimously perceived as a major responsibility of the mothers.

"I know my child is depending on me until six months ofage..." (Mo)

"It's just important that I give my wife good food as she is always giving breast milk to my child..." (Fa)

"I can do anything for my grandchild except giving breast milk.....it's a compulsory task of mothers....." (Gp)

However, the lack of skills in breastfeeding may hamper this important task. In the service provider's perspective, it was highlighted that the attachment of the infant to the breast is not practiced appropriately by the mothers which always results in feeding difficulties.

"Our mothers are always complaining of having no breast milk to feed the baby....but the problem is they are practicing poor feeding techniques..." (Sp)

\section{Theme 2. Maintaining hygiene}

Another main theme identified in this study was maintaining the hygiene of the infant including bathing the infant, washing the eyes and ears, hygiene of the perineum and care for the nails, which are viewed as utterly important aspects in parenting competencies. However, mothers often stated that they had no confidence to bathe the infant.

"I can do all the others, except bathing the baby....I feel fear to do that..." (Mo)

"My daughter never tries to bathe the baby...I've been bathing my grandson since his birth..." (Gp)

It was highlighted that the mothers are highly focused on maintaining the clean surroundings within the house and separate things used for the infant.

"I always keep the things used for my daughter separately like a basin, soap and other things..." (Mo)
According to the service provider's point of view, the mothers maintain hygienic practices and they have a good sense that poor hygienic practices lead to illnesses.

"Mothers try to maintain the hygienic practices at an optimum level as they can ..." (Sp)

\section{Theme 3. Dealing with crying}

Dealing with crying was also one of the parenting competency domains that emerged from the study.

According to the study, findings revealed that fathers are not satisfied with the mother's competencies in dealing with a crying infant.

"Sometimes (I) feel angry with the wife... she can't stop the baby from crying ..." (Fa)

"One day neighbors also came running to our home, when (they) heard continuous crying of the baby..." (Fa)

Mothers highlighted that they felt stressed during inconsolable crying of the infant and were completely depressed by feeling the discomfort of the infant.

"Really it's the hardest thing for me.......my mother is the only person who can deal with the crying.... even at midnight...." (Mo)

"My daughter also cries sometimes when it is difficult to calm down her son...." (Gp)

Service providers also experienced the poor ability of mothers in dealing with the crying of the infant.

"Mothers always get backward when their infants cry......always seeking help from others, never even trying ...." $(S p)$

\section{Theme 4. Dealing with illnesses}

Dealing with illnesses, especially the management of fever at home and preventing dehydration was another highlighted competency area in the study. The study findings highlighted that seeking medical advice is the most certain decision of the mothers just after the appearance of any ill health condition in the infant.

"When I feel the child is not well, I just visit the Doctor for taking medicine"(Mo) 
In contrast, some mothers had a good sense of prior home care when the infant is suffering from an illness.

"I have a thermometer at home and I can measure my baby's body temperature... If she is having fever, immediately I start sponging the baby using water and go to take medicine..." (Mo)

However, the service providers' perspectives indicated that some mothers had a poor sense of reporting the things that happened at home related to the illness of the child when they come to seek medical advice. It appears that this inability to report the features of illness accurately causes problems of interpreting the nature or severity of the illness.

"When I ask from the mother when her child got fever... or how many times her child vomited... some mothers were not confident enough to give an exactresponse...." (Sp)

Theme 5. Recognising the normal growth and development

Recognising the normal growth and development was another parenting competency domain that emerged from the study. Many mothers willingly took part in monitoring their children's growth.

"I always want to make sure that my baby is healthy and the baby is all right..." (Mo)

"Most of the mothers have a good insight to keep records in the Child Health Development Record.... (Sp)

However, mothers did not seem to possess the ability to interpret the information in the health records meaningfully. It was highlighted that most of the mothers had a tendency of comparing their child with others; especially children of their neighbors, relatives or friends, according to the service provider's perspective. This leads to uncertainty in mothers as to whether the growth and development of their child is appropriate.

"Mothers always compare the child with a known child and come to us with a lot of complaints.... but the child is ok..." (Sp)

\section{Discussion}

This study explored different dimensions of parenting competencies among mothers with infants at early infancy based on the views and experiences of mothers, fathers, grandparents and service providers. The findings highlighted that there are different domains of competencies among mothers. Also, both mothers and significant others in their inner family circle seem to perceive at least five main dimensions of competencies that are required by these mothers; namely, feeding the infant, maintaining hygiene, dealing with crying, dealing with illnesses and recognising the normal growth and development.

In interpreting the findings it was highlighted that most of the study participants who were interviewed were concerned that feeding the infant and maintaining the hygiene are foremost competency pillars in maternal parenting competencies. Further, it was noted that dealing with crying, dealing with illnesses and recognising normal growth and development were remarkably diverting competency domains towards elders of the mother and professional caregivers. Other studies revealed that prevention of infection, thermal protection, resuscitation of the newborn with asphyxia, exclusive breastfeeding, care of the low birth weight babies and identification and appropriate referral of sick neonates are leading pedigrees concerning maternal competencies $(14,15)$.

Another study found that the mother or caregiver is consistently responsive to the infants' care, meeting the infants' physical and psychological needs (3). As noted by Grover (7) protection (an environment that is safe from physical and emotional harm); good health (including safe water, hygiene); appropriate nutrition (including exclusive breastfeeding for the first six months), stimulation (opportunities to explore the world, express curiosity, engage in problem-solving) and language development (listening and responding) are important in describing the maternal parenting competencies.

The strengths of this study include the use of two different data collection techniques (FGD and IDI) with a wider circle of informants (mothers, fathers, grandparents, and senior community health and hospital staff). This approach facilitated the 
assembly of knowledge on parenting competencies at early infancy with comprehensive insight from different perspectives. All the interviews and discussions were conducted by the same investigator to minimise observer bias, which was an additional advantage. However, excluding participants who could not speak Sinhalese may hinder the generalisability of findings, although its effect would be minimal as nearly all of the ethnic groups participated in the study were well-conversant in spoken Sinhalese. However, as the cultural expectations of parenting competencies may vary across geographical boundaries, the replication of this study in diverse settings may further enhance the comprehensiveness of exploration.

Our findings suggest that a proper evaluation of parenting competencies is a vital requirement within the maternal and child care services in Sri Lanka. Many countries use validated tools to assess the parenting competencies (4). However, in Sri Lanka, no validated tools are available to assess this important aspect of family health. The findings of this study can be used to develop culturally specific tools to assess the level of maternal competencies by the local health system as well as to design educational interventions to enhance such competencies among the new mothers.

\section{Conclusions}

The study findings reflect that the major pillars concerning maternal parenting competencies are; feeding the infant, maintaining hygiene, dealing with crying, dealing with illnesses, and recognising the normal growth and development. In justifying the sense of optimal reliability of these findings, development of a culturally specific parenting competency assessment tool unique to Sri Lanka may prove fruitful. Evidence-based decisions arising from such tools can be utilized in setting priorities and establishing effective strategies to enhance parenting competencies in the identified domains through appropriate interventions at national level.

\section{References}

1. Emmanuel E, Creedy D, St. John W, Gamble J, Brown C. Maternal role development following childbirth among Australian women. Journal of Advanced Nursing, 2008; 64(1): 18-26.

2. Meleis A, Sawyer L, Im E Hilfinger, Messias D, Schumacher K. Experiencing transitions: An emerging middle-range theory. Advances in Nursing Science, 2000; 23(1): 12-28.

3. Mercer R. Nursing support of the process of becoming a mother. Journal of Obstetric, Gynecologic, and Neonatal Nursing, 2006; 35(5): 649-651.

4. Cardoso A, Silva AP. Representing nursing knowledge on maternal and neonatal health: A study on the cultural suitability of ICNP. International Nursing Review, 2010; 57(4): 426-434.

5. International Council of Nurses. 2011. International Classification of Nursing Practice. Lisbon.

6. Ribas JR, Bornstein M. Parenting knowledge: Similarities and differences in brazilian mothers and fathers. InterAmerican Journal of Psychology, 2005; 39(1): 5-12.

7. Grover D. (2005) The Young Child In The Family: Promoting Synergies Between Survival, Growth, And Development In Early Childhood. Presentation at the CARK MCH Forum. Dushambe, Tajikistan, September 20-22, 2005.

8. Hetherington E, Parker R, (Eds). 1999). Child Psychology: AContemporary Viewpoint (5 ${ }^{\text {th }}$ ed). McGraw-Hill; 1999.

9. Demographic and Health Survey 2006 - 2007. Department of Census and Statistics and Ministry of Healthcare \& Nutrition; 2009.

10. Family Health Bureau. Reproductive Health Management Information System. Colombo, Sri Lanka: Family Health Bureau Ministry of Health, Sri Lanka; 2010-11.

11. Fernando D, Gunawardene N, Weerasinghe M, Senerath U. (2011) Extent, Trends, and Determinants of Teenage Pregnancy in Three Districts of Sri Lanka, Karunaratne \& Sons (Pvt.) Ltd. Colombo.

12. Barlow A, Mullany B, Neault N, Compton S, Carter A, Hastings R, Billy T, Mescal VC, Lorenzo S, Walkup JT. Effect of a Paraprofessional Home-Visiting Intervention on American Indian Teen Mothers' and Infants' Behavioural Risks: A Randomized Controlled Trial. American Journal of Psychiatry, 2013; 170: 83-93. 
13. Gale, et al. Using the framework method for the analysis of qualitative data in multi-disciplinary health research. $B M C$ Medical Research Methodology, 2013; 13: 117.

14. World Health Organization. Essential Newborn Care Report of a Technical Working Group. Geneva: World Health Organization (WHO/FRH/MSM/96.13). Geneva: World Health Organization, 1996.

15. Costello A, Manandhar D, (Eds). Improving newborn health in developing countries. London: Imperial College Press; 1998. 